\title{
Persistent major alopecia following adjuvant docetaxel for breast cancer: incidence, characteristics, and prevention with scalp cooling
}

\author{
M. Martín ${ }^{1,6}$ - J. C. de la Torre-Montero ${ }^{2,3} \cdot$ S. López-Tarruella ${ }^{4} \cdot$ K. Pinilla ${ }^{5} \cdot$ A. Casado ${ }^{2} \cdot$ S. Fernandez ${ }^{2,4} \cdot$ Y. Jerez ${ }^{4}$. \\ J. Puente ${ }^{2} \cdot$ I. Palomero ${ }^{4} \cdot$ R. González del Val ${ }^{4} \cdot$ M. del Monte-Millan ${ }^{4} \cdot$ T. Massarrah $^{4} \cdot$ C. Vila ${ }^{2} \cdot$ B. García-Paredes ${ }^{2}$. \\ J. A. García-Sáenz ${ }^{2} \cdot$ A. Lluch ${ }^{5}$
}

Received: 11 June 2018 / Accepted: 13 June 2018 / Published online: 19 June 2018

(c) The Author(s) 2018

\begin{abstract}
Background Persistent alopecia (PA) after docetaxel has been recently described. The aim of our study is to establish the incidence and characteristics of PA following adjuvant docetaxel for breast cancer (BC) and to test the ability of scalp cooling in prevention.

Patients and methods BC patients receiving adjuvant chemotherapy followed or not by endocrine therapy (and a control group receiving only endocrine therapy) were interviewed in a single institution at 1.5 to 5 years following primary diagnosis searching for PA. A confirmatory prevalence study was later performed in other two institutions. Finally, a prevention study using prophylactic scalp cooling (PSC) with ELASTO-GEL hypothermia caps in patients receiving adjuvant docetaxel was performed.

Results In the initial prevalence study (492 patients), minor forms of PA (grade 1) were recorded with all chemotherapy regimens and aromatase inhibitors. Patients receiving docetaxel regimens at cumulative dose (CD) $\geq 400 \mathrm{mmg} / \mathrm{m}^{2}$ presented a significantly higher prevalence of grades 1 PA (33-52\%) and 2 PA (5-12\%). Prevalence of grade 2 PA with docetaxel $\mathrm{CD} \geq 400 \mathrm{mmg} / \mathrm{m}^{2}$ was confirmed in two other institutions. Overall, grade 2 PA was seen in $10.06 \%$ (95\% CI 7.36-13.61) of 358 patients with docetaxel regimens reaching $\mathrm{CD} \geq 400 \mathrm{mmg} / \mathrm{m}^{2}$, but not in patients with lower docetaxel $\mathrm{CD}$, other chemotherapy regimens, or endocrine therapy alone. In prevention trial, no grade 2 PA occurred among 116 patients receiving adjuvant docetaxel $\left(\geq 400 \mathrm{mmg} / \mathrm{m}^{2}\right)$ and PSC followed-up after a 96 months median time. PSC was well tolerated. No scalp relapses were seen among 30 patients ( $22 \%$ of all inclusions) having disease relapse.

Conclusion Adjuvant treatment with docetaxel $\left(\mathrm{CD} \geq 400 \mathrm{mmg} / \mathrm{m}^{2}\right)$ is associated with a significant rate of grade $2 \mathrm{PA}$, leading to wearing a wig, in around $10 \%$ of patients. This toxicity was completely prevented with scalp cooling. Clinical Trial Reference: NCT00515762.
\end{abstract}

Keywords Alopecia $\cdot$ Breast cancer $\cdot$ Docetaxel $\cdot$ Scalp cooling

Electronic supplementary material The online version of this article (https://doi.org/10.1007/s10549-018-4855-2) contains supplementary material, which is available to authorized users.

\section{Martín}

mmartin@geicam.org

1 Medical Oncology Service, Hospital General Universitario Gregorio Marañón, Instituto de Investigación Sanitaria Gregorio Marañón, CIBERONC, GEICAM, Universidad Complutense, Madrid, Spain

2 Medical Oncology Department, Hospital Clínico San Carlos, Instituto de Investigación Sanitaria del Hospital Clínico San Carlos (IdISSC), CIBERONC, Madrid, Spain
3 Universidad Pontificia Comillas, Madrid, Spain

4 Medical Oncology Service, Hospital General Universitario Gregorio Marañón, Instituto de Investigación Sanitaria Gregorio Marañón, CIBERONC, Madrid, Spain

5 Medical Oncology Department, Hospital Clínico Universitario, CIBERONC, Valencia, Spain

6 Hospital General Universitario Gregorio Marañón, Medical Oncology Service, Calle Maiquez, no. 9, 28007 Madrid, Spain 


\section{Introduction}

Alopecia is a common toxicity of anticancer drugs and is considered by the patient as the second worst sideeffect of chemotherapy, after emesis [1]. Cytotoxic agents cause alopecia by inhibiting the mitotic process in anagen hair roots. Hair loss usually starts 2 weeks following the initiation of chemotherapy, and has been traditionally considered to be reversible in all cases, after 3-4 months of cessation of therapy [2]. However, the observation of persistent alopecia (PA) long time after the end of adjuvant docetaxel chemotherapy in some of our BC patients, prompted us to study the prevalence of this phenomenon in $\mathrm{BC}$ patients and to explore the ability of scalp hypothermia to prevent this toxicity.

\section{Materials and methods}

The main aims of the study were the following:

1. To establish the prevalence of PA resulting from adjuvant docetaxel for BC.

2. To test the efficacy of scalp hypothermia in preventing docetaxel-associated PA.

In the prevalence study, the population consisted of consecutive BC patients seen in the outpatient clinics of a Spanish institution (Hospital Clinico San Carlos, Madrid, HCSC) using Taxotere (Sanofi-Aventis, Paris). In order to confirm the prevalence of PA after docetaxel, the study was extended later on to other two institution (Instituto de Investigación Sanitaria Gregorio Marañón, Madrid, IISGM and Hospital Clínico de Valencia, HCUV) in which several docetaxel generics were used. Selection criteria were the following:

1. BC patients with UICC stage I-III.

2. Patients on follow-up 1.5-5 years after adjuvant/neoadjuvant anthracycline or taxane chemotherapy, alone or followed by endocrine adjuvant therapy and/or trastuzumab.

3. No evidence of disease recurrence.

Alopecia was graded according to the criteria used in the Common Terminology Criteria for Adverse Events CTCAE v3.0 for acute (reversible) alopecia. Grade 1 PA was defined as weakening of hair or partial alopecia and not leading to the use of a wig, after at least 18 months from the end of adjuvant chemotherapy (ACT). Grade 2 PA was defined as complete alopecia that requires a wig after at least 18 months from the end of ACT. In the first institution (HCSC), both grade 1 and 2 PA were recorded and a subset of 144 patients treated with adjuvant endocrine aromatase therapy (without chemotherapy) was also included to ascertain the contribution of this therapy to PA. In the two other institutions, only grade 2 PA was recorded.

Patient (age, menopausal status), tumor (stage, hormone receptors, her2/neu status), and treatment characteristics (chemotherapy schedules, radiation, endocrine treatment, trastuzumab) and time from surgery and from conclusion of adjuvant therapy were recorded. Patients were interviewed and explored to detect PA.

Patients with PA in one institution (HCSC) were examined by a dermatologist who analyzed the pattern of alopecia and performed a $4 \mathrm{~mm}$ punch biopsy of scalp for microscopic examination.

\section{Prevention study}

The prevention study (NCT00515762) was carried out in one of the participating institutions (HCSC) in a series of consecutive patients receiving one of the two following adjuvant regimens:

(1) $\mathrm{AC} / \mathrm{EC} \rightarrow \mathrm{T}$ : doxorubicin $\left(60 \mathrm{mg} / \mathrm{m}^{2}\right)$ or epirubicin $\left(90 \mathrm{mg} / \mathrm{m}^{2}\right)$ in combination with cyclophosphamide $\left(600 \mathrm{mg} / \mathrm{m}^{2}\right)$ for 4 cycles followed by docetaxel (100 mg/m $\mathrm{m}^{2} \times 4$ cycles $)$.

(2) TAC: docetaxel $75 \mathrm{mg} / \mathrm{m}^{2}$, doxorubicine $50 \mathrm{mg} / \mathrm{m}^{2}$, cyclophosphamide $500 \mathrm{mg} / \mathrm{m}^{2}, \mathrm{IV}$, every 3 weeks $\times 6$ cycles.

Taxotere ${ }^{\circledR}$ (Sanofi-Aventis, Paris) was the only docetaxel used in this trial. Patients receiving $\mathrm{AC} / \mathrm{EC} \rightarrow \mathrm{T}$ started prophylactic hypothermia in the docetaxel part of chemotherapy. Patients receiving TAC chemotherapy had scalp hypothermia from the first cycle on.

A frozen hypothermic scalp cap (Elasto-gel) maintained at stable $-25{ }^{\circ} \mathrm{C}$, was placed on the patient scalp $15 \mathrm{~min}$ before docetaxel administration, and replaced by a second one 45 min later. The ELASTO-GEL hypothermia cap is a medical device available in Europe (European Directive 93/42/EEC) and US, (Icewraps, Escondido, CA, USA) made of a seamless flexible material containing a glycerin-based hydrogel that remains flexible even at very low temperatures $\left(-30{ }^{\circ} \mathrm{C}\right)$. The cap supplies local cooling and its use is indicated to prevent chemotherapy-induced alopecia in patients with cancer.

The cap was maintained until 30 min after the end of docetaxel infusion. This procedure was repeated each time the docetaxel treatment was administered until the end of chemotherapy. Before the first infusion of chemotherapy, 
a baseline assessment of the degree of alopecia was conducted. Cap comfort and tolerability were assessed $1 \mathrm{~h}$ after completing each infusion of the treatment. Follow-up visits took place every 6 months after treatment has ended in order to determine the percentage of patients with persistent alopecia.

Sample calculation was based on the results of the initial prevalence study carried out at the HCSC, in which around $50 \%$ of patients receiving TAC or anthracyclines followed by docetaxel without any method of prevention had grade 1 or 2 PA. According to the previous bibliography [3], we hypothesized that scalp hypothermia will reduce the alopecia rate to $20 \%$. To achieve a potency of $99 \%$ for detecting differences with the null hypothesis comparator ( $H o: p=0.50)$, using a bilateral Chi-squared test with a 5\% level of significance $($ alpha $=0.05), 41$ fully evaluable patients were required. Considering that evaluable patients should have received a cumulative docetaxel dose of $\geq 400 \mathrm{mg} / \mathrm{m}^{2}$, an estimated drop-out rate of $30 \%$ over the study course was expected (including patients stopping therapy or with significant dose reductions) and, therefore, 59 patients were estimated to be included to obtain 41 patients for full assessment.

In 2008, after the enrolment of 59 patients, it was evident that the statistical assumption of the trial (incidence of PA with scalp hypothermia of 20\%) was erroneous, since no single patient presented this side effect with scalp hypothermia. No significant side effects with ELASTO-GEL had been recorded either. Therefore, the protocol was amended to increase the trial sample size to 120 evaluable patients, to have a better estimation of the efficacy and tolerance of the method. A final sample size of 150 patients (including dropouts) was then established.

The patients were follow-up every 6 months during the first 5 years and yearly afterwards in order to evaluate scalp hair recovery.

The studies were approved by the Ethical Committees of the participating institutions. Patients signed an informed consent before being enrolling. Pictures of alopecia were reproduced (enmasked) with permission of the patients.

\section{Results}

\section{Prevalence study}

\section{Clinical findings}

The prevalence study was initially performed at HCSC between December 2005 and May 2006. 492 patients (58\% postmenopausal, $42 \%$ premenopausal, median age 53 years)

Table 1 Prevalence study

\begin{tabular}{ll}
\hline Number of patients & 492 \\
Median age (years, range) & $53(26-76)$ \\
Menopausal status & $58 \%$ \\
Postmenopausal & $42 \%$ \\
Premenopausal & \\
Adjuvant therapy & $0 / 148$ \\
FAC/FEC/AC/EC $\times 6$ cycles & $0 / 29$ \\
FAC/FEC/AC/EC $\times 4$ cycles $\rightarrow$ weekly paclitaxel $\times 8-12$ cycles & $0 / 31$ \\
Epirubicin $90 \mathrm{mg} / \mathrm{m}^{2}$ plus docetaxel $75 \mathrm{mg} / \mathrm{m}^{2} \times 4$ cycles $\rightarrow$ Capecitabine $\times 4$ cycles & 0 \\
TC $\times 4$ cycles & 0 \\
TC $\times 6$ cycles & $4 / 74$ \\
TAC $\times 6$ cycles & $(5.4 \%)$ \\
TCH $\times 6$ cycles & 0 \\
A/E $+/-C \times 4$ cy $\rightarrow$ docetaxel $100 \mathrm{mg} / \mathrm{m}^{2} \times 4$ cycles $+/-$ trastuzumab & $8 / 66$ \\
Tamoxifen & $(12 \%)$ \\
Aromatase inhibitors & \\
\hline
\end{tabular}

Patients/treatment characteristics

HCSC Hospital Clinico San Carlos, Madrid; FAC 5-fluorouracil, doxorubicin, cyclophosphamide; FEC, 5-fluorouracil, doxorubicin, cyclophosphamide; AC/EC, doxorubicin or epirubicin plus cyclophosphamide; $T C$ docetaxel $75 \mathrm{mg} / \mathrm{m}^{2}$ plus cyclophosphamide; TAC, docetaxel $75 \mathrm{mg} / \mathrm{m}^{2}$, doxorubicin, cyclophosphamide; $T C H$, docetaxel $75 \mathrm{mg} / \mathrm{m}^{2}$, carboplatin AUC 6, trastuzumab

${ }^{\mathrm{a}}$ Without prior adjuvant chemotherapy

${ }^{\mathrm{b}}$ Without prior adjuvant chemotherapy; some patients included in this group have received tamoxifen followed by an aromatase inhibitor 
Fig. 1 Persistent alopecia (PA) in patients treated with chemotherapy or endocrine therapy in one of the institutions (HCSC). TAM: tamoxifen; AI: aromatase inhibitors (no chemotherapy); FAC/FEC: 5-fluorouracil,doxorubicin or epirubicin, cyclophosphamide $\times 6$ cycles; ANT-PACL: anthracyclines $\times 4$ cycles followed by paclitaxel; ETCAP: epirubicin plus docetaxel $75 \mathrm{mg} / \mathrm{m} 2 \times 4$ cycles, followed by capecitabine; TAC: docetaxel $75 \mathrm{mg} / \mathrm{m}^{2}$, doxorubicin, cyclophosphamide $\times 6$ cycles; ANT-DOCE: anthracyclines $x$ 4 cycles followed by docetaxel $100 \mathrm{mg} / \mathrm{m}^{2} \times 4$ cycles; Around two-thirds of the patients treated with adjuvant chemotherapy received endocrine therapy with tamoxifen, aromatase inhibitors, or both afterwards

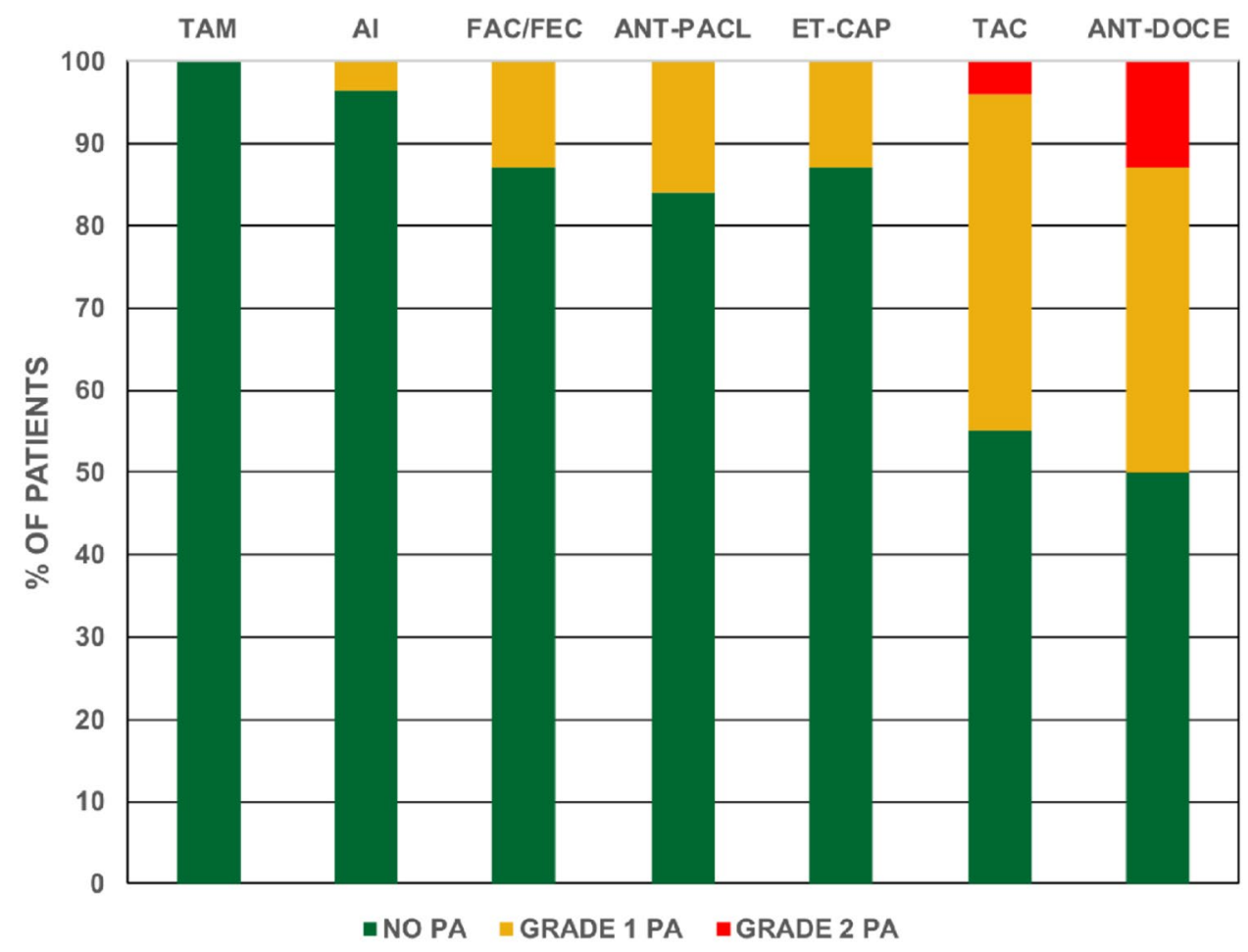

were interviewed and explored (Table 1). Grade 1 PA was found with all chemotherapy regimens (variable proportions) and aromatase inhibitors (3.5\% of cases), but not with tamoxifen (Fig. 1). Patients treated with docetaxel regimens (cumulative dose $(\mathrm{CD}) \geq 400 \mathrm{mmg} / \mathrm{m}^{2}$ ) presented with a significantly higher prevalence of grade 1 PA (33-52\%) and were the only ones having grade $2 \mathrm{PA}(5-12 \%)$. The prevalence grade $2 \mathrm{PA}$ with docetaxel was confirmed at two additional Spanish institutions. These institutions joined the prevalent study later, to confirm the prevalence of grade 2 PA (IISGM Dec 2009-August 2012 and HCV March 2014-August 2014). Minor forms of PA (grade 1) were not collected, since they were more difficult to agree upon in a homogenous way. None of the interviewed patients had scalp hypothermia during chemotherapy administration.

Taking together the three institutions, grade 2 PA was seen in patients receiving docetaxel regimens with a $\mathrm{CD} \geq 400 \mathrm{mmg} / \mathrm{m}^{2}$ (36/358, 10.06\%, 95\% CI 7.36-13.61), but not in 59 patients with docetaxel regimens reaching lower CD of the drug $\left(300 \mathrm{mg} / \mathrm{m}^{2}\right)$, or chemotherapy regimens not containing docetaxel $(n=306)$. Weekly paclitaxel was not associated with grade 2 PA. The incidence of docetaxel-induced grade 2 PA was similar in patients with $(22 / 221,9.96 \%)$ and without hormonal therapy (14/137, $10.2 \%$ ). Trastuzumab did not increase the incidence of docetaxel-induced PA (Table $1 \mathrm{~b}$ in the Supplement). The median follow-up of patients following the conclusion of chemotherapy was 43 months (range 18-60 months).
Clinically, patients with grade 2 PA showed a practically complete loss of hair density (Fig. 2, panels a to i) and reported no significant improvement of hair growth over time, after a median follow-up of 48 months. Ten of these patients from HCSC have been followed up now for more than 10 years and did not show any sign of hair recovery in spite of the use of multiple dermatological therapies (including topical minoxidil at $5 \%$ in all cases).

\section{Pathology findings}

Punch biopsy specimens were obtained from 10 patients with PMA treated with AC followed by docetaxel or with TAC at HCSC. All samples showed a marked reduction in large, terminal hairs with a reciprocal increase in small, vellus-like hairs (miniaturization of follicles); features very similar to those observed in androgenic alopecia. Neither significant peri-follicle inflammation nor fibrosis was noted (Fig. 3, in the Supplement).

\section{Prevention study: scalp hypothermia}

Between March 2006 and September 2009, 136 operable $\mathrm{BC}$ patients scheduled to receive docetaxel at $\mathrm{CD} \geq 400 \mathrm{mg} /$ $\mathrm{m}^{2}$, either as a combination regimen (TAC) or as a single agent after four courses of doxorubicin/epirubicine plus cyclophosphamide, consented to enter the prevention study in HCSC. Patient characteristics are shown in Table 2. 
Fig. 2 Persistent alopecina after 46 to 120 months following the conclusion of docetaxel chemotherapy. Panels a-i: Grade 2 Persistent Alopecia. Panels j-l: Grade 1 Persistent Alopecia (with permission from the patients)
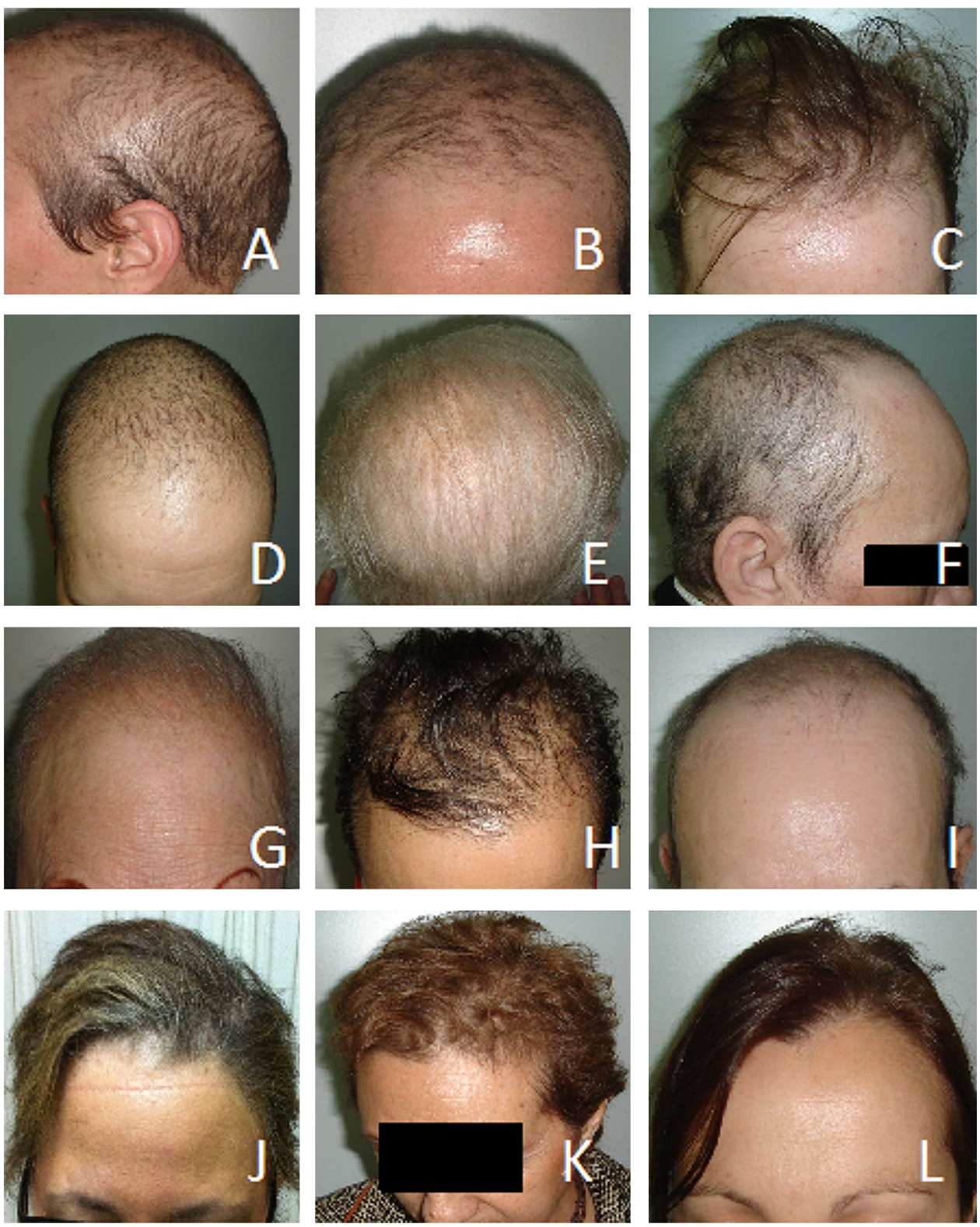

One hundred and twenty patients received docetaxel $\mathrm{CD} \geq 400 \mathrm{mg} / \mathrm{m}^{2}$ and were considered evaluable for study purposes. The remaining patients did not reach the threshold docetaxel dose due to docetaxel interruption (6 patients) or dose reductions (10 patients).

After a median follow-up of 8 years, only a single patient among those evaluable presented with grade $1 \mathrm{PA}$ $(0.8 \%, 95 \%$ CI $0.15-4.56 \%)$. She was a 50 year old patient treated with AC followed by a CD of docetaxel of $400 \mathrm{mg} /$ $\mathrm{m}^{2}$, followed by 5 years of letrozol. No cases of grade 2 PA were recorded. None of 16 non-evaluable patients (docetaxel dose $<400 \mathrm{mg} / \mathrm{m}^{2}$ ) presented any kind of PA.

$\mathrm{BC}$ relapses were seen in 30 patients (22\% of all inclusions). Main sites of relapses were preserved breast $(n=1)$, locoregional lymph nodes $(n=4)$, bone $(n=16)$, and visceral relapses $(n=9)$. No scalp relapses were observed, neither isolated nor in association with other metastatic sites.

The tolerance to ELASTO-GEL device was good. Thirteen patients $(10 \%)$ complained of mild headache, but all could finish the scheduled procedure.

\section{Discussion}

Our study showed that PA is a significant problem for BC patients who have had received docetaxel-containing regimens; a significant percentage of patients is affected, and with no signs of recovery even after several years of followup. Alopecia is one of the most distressing side-effects of 
Table 2 Intervention study (scalp hypothermia)

\begin{tabular}{ll}
\hline Characteristics and treatment & Number of patients (\%) \\
\hline Total number enrolled & $136(100 \%)$ \\
Menopausal status & \\
Premenopausal & $57(41.9 \%)$ \\
Postmenopausal & $79(58.1 \%)$ \\
Age (years) & \\
Median & 53 \\
Range & $28-84$ \\
Chemotherapy régimen & \\
AC/EC $\rightarrow$ docetaxel & $90(66.2 \%)$ \\
TAC & $46(33.8 \%)$ \\
Actual docetaxel cumulative dose administered (mg/m $\left.{ }^{2}\right)$ & \\
Range & $160-450$ \\
$<400$ & $16(11.8 \%)$ \\
400 & $81(59.6 \%)$ \\
$>400$ & $39(28.7 \%)$ \\
Docetaxel dose modifications & \\
Reduction & $10(7.4 \%)$ \\
Interruption & $6(4.4 \%)$ \\
Adjuvant trastuzumab & \\
Yes & \\
No & \\
Adjuvant hormonal therapy & $45(38.8 \%)$ \\
Tamoxifen $(+/-$ LHRH analogs) & $71(61.2 \%)$ \\
Aromatase inhibitors $(+/-$ tamoxifen+/-LHRH analogs) & \\
None & $37(27 \%)$ \\
\hline & $49(36 \%)$ \\
\hline
\end{tabular}

Patients characteristics and treatment
$\mathrm{ACH}$ and has been traditionally considered to be reversible at the conclusion of treatment BC [2]. Only a few cases of PA after chemotherapy had being described in the past, usually following high-dose chemotherapy with bone marrow or peripheral stem cell support $[4,5]$.

Several randomized phase III trials conducted during the decade of the 90 s had shown than taxane-containing regimens are superior to classical anthracycline-containing chemotherapy [6, 7] and, therefore, these regimens have been considered the standard $\mathrm{ACT}$ for $\mathrm{BC}$ in most countries.

However, many of the taxane-containing regimens are also more toxic than the older regimens. The acute toxicity of docetaxel to skin and skin annexes have been described in previous clinical trials, and include erythema and skin desquamation of the limbs and nail changes in a considerable proportion of patients [8-10]. The skin toxicity incidence in phase III trials with $100 \mathrm{mg} / \mathrm{m}^{2}$ docetaxel doses ranged from 32 to $37 \%$, with 4 to $4.5 \%$ of patients having grade $3-4$ toxicity. Additionally, nearly $40 \%$ of patients had nail disorders.

Significant alopecia had been reported in $74.2 \%$ of docetaxel-treated patients [10], but had been described as systematically reversible. In 2001, Nabholtz et al. described, for the first time, four patients with long-lasting ( $>2$ years)
PA following TAC (docetaxel, doxorubicin, cyclophosphamide) chemotherapy for metastatic BC [11], the finding having generated little attention at that time. In 2006, Sedlacek reported, the phenomenon of PA following ACT for $\mathrm{BC}$ [12]. The study retrospectively reviewed hair recovery in 496 consecutive patients who, over a period of 11 years, had been treated with ACT. All patients had at least one year of follow-up after therapy. The average time from the last chemotherapy dose was 48 months (range 19-85 months). The study defined PA as hair re-growth of $<50 \%$ of the prechemotherapy amount of hair, as judged by both the patient and the investigator (similar to grade 2 alopecia according to CTCAE v4.0). The chemotherapy regimens in this study were classified in 3 categories: group A (doxorubicin regimens without taxanes); group B (regimens including doxorubicin + paclitaxel); and group C (regimens including doxorubicin + docetaxel: AC followed by docetaxel, doxorubicin + docetaxel, TAC, AC followed by docetaxel + capecitabine, docetaxel + doxorubicin followed by CAF, CAF followed by docetaxel). No PA was found in groups 1 and 2 , whereas it was present in 6.3\% (7/112) of patients in group C. All patients with persistent significant alopecia had received AC followed by docetaxel $\left(100 \mathrm{mg} / \mathrm{m}^{2}\right)$. 
Our study showed that grade $1 \mathrm{PA}$ is present in a small proportion of patients following most regimens of ACT and/or aromatase inhibitors, the majority of them in postmenopausal patients. Progressive spontaneous alopecia is not an infrequent finding in postmenopausal women. Medications, concomitant chronic diseases, telogen effluvium, and, mostly, female pattern hair loss (FMHL) are frequent causes of alopecia in women post-menarche [13]. Aromatase inhibitors have also been reported as a cause of male pattern hair loss [14] and this was also seen in our study. Although causes other than chemotherapy itself can be involved in PA, the high proportion of patients having grade 1 PA (35-50\%) after regimens including docetaxel at $\mathrm{CD} \geq 400 \mathrm{mg} / \mathrm{m}^{2}$ suggests an ethiologic role for this drug. Besides, we found grade 2 PA only in patients treated with docetaxel regimens achieving the same cumulative dose of the drug being recorded in $10.06 \%$ of this population $(95 \%$ CI 7.36-13.61\%).

The pathogenesis of PMA following docetaxel is not known. Our study suggests that docetaxel can cause permanent damage of hair follicles. Punch biopsy specimens obtained from 10 patients with PMA showed an unspecific pattern of androgenic-like alopecia, with marked reduction in large, terminal hairs.

Kluger et al. [15] reported 21 Caucasian BC patients who presented PA after docetaxel adjuvant therapy, showing a moderate or intense androgenetic-like pattern of scalp alopecia. PA was defined as absent or incomplete hair regrowth at $\geq 6$ months post chemotherapy. This description probably correspond to cases of grade 1 or 2 PA. Biopsy specimen examinations were normal or displayed the androgeneticlike pattern, as in our study. The chemotherapy protocols included docetaxel at CD of $300 \mathrm{mg} / \mathrm{m}^{2}$ (12 patients) or $\geq 400 \mathrm{mg} / \mathrm{m}^{2}$ ( 8 cases). Fonia et al. [16] described 10 patients with persistent alopecia following different combination regimens including docetaxel. The histopathologic study showed features of non-scarring alopecia with hair follicle units preservation, reduced hair density, and increased vellus hair follicles, a pattern very similar to that seen in our patients.

In recent years, renewed interest in chemotherapyinduced hair loss prevention has arised. Two recent trials have supported the ability of scalp hypothermia to reduce or completely prevent chemotherapy-induced alopecia [17, 18]. However, these trials were aimed at preventing chemotherapy-induced acute (reversible) alopecia. The aim of our preventive study was different, the prevention of PA following docetaxel. The rationale of our study was based on the success of chilled gloves in reducing nail and cutaneous toxicity of the hand associated with docetaxel therapy [19].

Our study showed that scalp cooling is very effective in preventing persistent alopecia following docetaxel. No patient experienced grade $2 \mathrm{PA}$ and only one patient $(0.8 \%$, $95 \%$ CI $0.15-4.56 \%$ ) had grade 1 PA.

A concern that has reduced in the past the use of scalp hypothermia for preservation of hair during chemotherapy is the risk of scalp metastases. The reduced exposition of the scalp to docetaxel or other chemotherapy drugs could facilitate the persistence of tumor cells, leading to scalp metastases months to years later [20]. However, a recent meta-analysis has found that the incidence of scalp metastases is very low, regardless of scalp cooling: $0.61 \%$ (95\% CI $0.32-1.1 \%$ ) with scalp cooling versus $0.41 \%$ (95\% CI $0.13-0.94 \%)$ in a control group $(p=0.43)$ [21]. Therefore, scalp cooling is unlikely to be a cause of increase incidence of scalp metastases.

In conclusion, PA following adjuvant docetaxel chemotherapy is a significant toxicity of the drug, particularly when administered at $\mathrm{CD}$ of $\geq 400 \mathrm{mg} / \mathrm{m}^{2}$. Scalp cooling is very effective in preventing this undesirable side effect of docetaxel.

Funding MM, AL, and JAGS have received funding from Centro de Investigación Biomédica en Red in the area of Breast Oncology of the Instituto de Salud Carlos III (CIBERONC).

\section{Compliance with ethical standards}

Conflict of interest The authors declare that they have no conflicts of interest.

Ethical approval The authors declare that the experiments comply with the current laws of the country in which it was performed.

Open Access This article is distributed under the terms of the Creative Commons Attribution 4.0 International License (http://creativeco mmons.org/licenses/by/4.0/), which permits unrestricted use, distribution, and reproduction in any medium, provided you give appropriate credit to the original author(s) and the source, provide a link to the Creative Commons license, and indicate if changes were made.

\section{References}

1. Coates A, Abraham S, Kaye SB et al (1983) On the receiving endpatient perception of the side-effects of cancer chemotherapy. Eur J Cancer Clin Oncol 19:203-208

2. Hood AF (1986) Cutaneous side effects of cancer chemotherapy. Med Clin N Am 70:187-209

3. Katsimbri P, Bamias A, Pavlidis N (2000) Prevention of chemotherapy-induced alopecia using an effective scalp cooling system. Eur J Cancer 36:766-771

4. Baker BM, Wilson CL, Davis AL et al (1991) Busulfan/cyclophosphamide conditioning for bone marrow transplantation may lead to failure to hair re-growth. Bone Marrow Transpl 7:43-47

5. Tosti A, Piraccini BM, Vicenzi C et al (2005) Permanent alopecia after busulfan chemotherapy. Br J Dermatol 152:1056-1058

6. Henderson C, Berry D, Demetri G et al (2003) Improved outcomes from adding sequential paclitaxel but not from escalating 
doxorubicin dose in an adjuvant chemotherapy regimen for patients with node-positive primary breast cancer. J Clin Oncol 21:976-983

7. Martin M, Pienkowski T, Mackey J et al (2005) Adjuvant docetaxel for node-positive breast cancer. N Engl J Med 352:2302-2313

8. Childress J, Lokich J (2003) Cutaneous hand and foot toxicity associated with cancer chemotherapy. Am J Clin Oncol $26: 435-436$

9. Minisini AM, Tosti A, Sobrero AF et al (2003) Taxane-induced nail changes: incidence, clinical presentation and outcome. Ann Oncol 14:333-337

10. Calhoun EA, Welshman E, Brown S (2005) Taxane-induced alopecia: a historical review of patients with ovarian or breast cancer treated with paclitaxel, docetaxel or paclitaxel poliglumex. J Clin Oncol 23(June 1 suppl):740 s

11. Nabholtz JM, Mackey JR, Smylie M (2001) Phase II study of docetaxel, doxorubicin, and cyclophosphamide as first-line chemotherapy for metastatic breast cancer. J Clin Oncol 19:314-321

12. Sedlacek SM (2006) Persistent significant alopecia (PSA) from adjuvant docetaxel after doxorubicin/cyclophosphamide (AC) chemotherapy in women with breast cancer. Breast Cancer Res Treat 100(suppl1):s116

13. Roberts WE (2006) Dermatologic problems of older women. Dermatol Clin 24:271-280

14. Rossi A, Iorio A, Scali E et al (2013) Aromatase inhibitors induce "male pattern hair loss" in women? Ann Oncol 24:1710-1711

15. Kluger N, Jacot W, Frouin E (2012) Permanent scalp alopecia related to breast cancer chemotherapy by sequential fluorouracil/ epirubicin/cyclophosphamide (FEC) and docetaxel: a prospective study of 20 patients. Ann Oncol 23:2879-2884

16. Fonia A, Cota C, Setterfield F, Goldberg LJ, Fenton DA, Stefanato CM (2017) Permanent alopecia in patients with breast cancer after taxane chemotherapy and adjuvant hormonal therapy: clinicopathologic findings in a cohort of 10 patients. J Am Acad Dermatol 76:948-957

17. Rugo HS, Klein P, Melin SA et al (2017) Association between use of a scalp cooling device and alopecia after chemotherapy for breast cancer. JAMA 317:606-614

18. Nangia J, Wang T, Osborne C et al (2017) Effect of a scalp cooling device on alopecia in women undergoing chemotherapy for breast cancer. The SCALP randomized clinical trial. JAMA 317:596-605

19. Scotté F, Tourani JM, Banu E et al (2005) Multicenter study of a frozen glove to prevent docetaxel-induced onycholysis and cutaneous toxicity of the hand. J Clin Oncol 23:4424-4429

20. Lemieux J, Desbiens C, Hogue JC (2011) Breast cancer scalp metastasis as first metastatic site after scalp cooling: two cases of occurrence after 7- and 9-year follow-up. Breast Cancer Res Treat 128(2):563-566

21. Rugo HS, Mellin SA, Voigt J (2017) Scalp cooling with adjuvant/neoadjuvant chemotherapy for breast cancer and risk of scalp metastases: systematic review and meta-analysis. Breast Cancer Res Treat 163:199-205 